FACTA UNIVERSITATIS (NIŠ)

Ser. Math. Inform. Vol. 35, No 4 (2020), 949-961

https://doi.org/10.22190/FUMI2004949Y

\title{
SOME NOTES ON KENMOTSU MANIFOLD
}

\author{
Halil İbrahim Yoldaş and Erol Yaşar
}

(C) 2020 by University of Niš, Serbia | Creative Commons Licence: CC BY-NC-ND

Abstract. In the present paper, we will deal with a Kenmotsu manifold M. Firstly, we will study the notion of torse-forming vector field on such a manifold. Then, we will investigate some curvature conditions such as $Q . \mathcal{M}=0$ and $C . Q=0$ on such a manifold and obtain some necessary conditions for such a manifold given as to be Einstein. Also, we will study a Kenmotsu manifold $M$ admitting a Ricci soliton and give an example for this manifold.

Keywords: Kenmotsu manifold; torse-forming vector field; Einstein manifold; Ricci soliton.

\section{Introduction}

A Riemannian manifold $(M, g)$ is called a Ricci soliton if there exists a constant $\lambda \in \mathbb{R}$ and a vector field $V \in \Gamma(T M)$ such that

$$
\left(£_{V} g\right)(X, Y)+2 S(X, Y)+2 \lambda g(X, Y)=0,
$$

where $£_{V} g$ denotes the Lie-derivative of the metric tensor $g$ along vector field $V$, $S$ is the Ricci tensor of $M$ and $X, Y$ are arbitrary vector fields on $M$. If $£_{V} g=0$ and $£_{V} g=\rho g$, then potential vector field $V$ is said to be Killing and conformal Killing, respectively, where $\rho$ is a function. Also, when $V$ is zero or Killing in (1.1), then the Ricci soliton reduces to Einstein manifold. So, it is considered as a natural generalization of Einstein metric. In addition, a Ricci soliton is called a gradient if the potential vector field $V$ is the gradient of a potential function $-f$ (i.e., $V=-\nabla f)$ and is called shrinking, steady or expanding depending on $\lambda<0, \lambda=0$ or $\lambda>0$, respectively.

The notion of Ricci soliton in Riemannian geometry was introduced by Hamilton in 1988 [11]. This notion corresponds to the self-similar solution of Hamilton's Ricci

Received October 25, 2019; accepted December 31, 2019

2020 Mathematics Subject Classification. Primary 53C15; Secondary 53C25, 53D15. 
flow: $\frac{\partial}{\partial t} g=-2 S$, viewed as a dynamical system on the space of Riemannian metrics modulo diffeomorphims and scaling. Also, Ricci solitons model the formation of singularities in the Ricci flow. In the framework of the contact geometry, they have been studied by many mathematicians in some different classes of contact geometry since Sharma applied Ricci solitons to K-contact manifolds [20]. For the recent studies on Ricci solitons, we refer to ([1], [7], [9], [10], [15], [17], [21], [24] and [25]).

On the other hand, torse-forming vector fields were firstly defined and studied by Yano [22]. They appear in many areas of differential geometry and physics. In recent years, they were studied by different authors such as Blaga et al. [2], Crasmareanu [8], Mandal et al. [14], Mihai et al. [16] and many others. According to Yano, a vector field $v$ on a Riemannian manifold $(M, g)$ is called torse-forming if it satisfies the following condition

$$
\nabla_{X} v=f X+\alpha(X) v
$$

for any $X \in \Gamma(T M)$, where $\nabla$ is the Levi-Civita connection on $M, \alpha$ is a $1-$ form and $f$ is a smooth function on $M$. If the 1 -form $\alpha$ vanishes identically in (1.2), the vector field $v$ is called a concircular [6]. If $\alpha=0$ and $f=1$ in (1.2), then $v$ is called a concurrent vector field [5]. If $f=-1$ in (1.2), then $v$ is called an irrotational vector field [2]. Also, the vector field $v$ is called a recurrent if it satisfies (1.2) with $f=0$.

The paper is organized as follows:

Section 1 is concerned with introduction. In section 2, we give some basic notions which are going to be needed. In section 3, we consider a Kenmotsu manifold $M$ endowed with a torse-forming vector field $v$ and find that the vector field $v$ is a pointwise collinear with the structure vector field $\xi$. In section 4 , we study a Kenmotsu manifold $M$ under some curvature conditions and deal with Ricci solitons on such a manifold. Also, we give an example to support our results.

\section{Preliminaries}

In this section, we shall give a brief review of some fundamental definitions and formulas of almost contact metric manifolds from [3], [13] and [23].

A $(2 n+1)$-dimensional smooth manifold $M$ is an almost contact metric manifold with an almost contact metric structure $(\varphi, \xi, \eta, g)$ such that $\varphi$ is a tensor field of type $(1,1), \xi$ is a vector field (called the characteristic vector field) of type $(0,1)$, 1 - form $\eta$ is a tensor field of type $(1,0)$ on $M$ and the Riemannian metric $g$ satisfies the following relations:

$$
\begin{aligned}
\varphi^{2} X & =-X+\eta(X) \xi \\
\eta(\xi) & =1, \\
\varphi \xi & =0 \\
\eta \circ \varphi & =0
\end{aligned}
$$


and

$$
\begin{aligned}
g(\varphi X, \phi Y) & =g(X, Y)-\eta(X) \eta(Y) \\
g(\varphi X, Y) & =-g(X, \varphi Y) \\
\eta(X) & =g(X, \xi)
\end{aligned}
$$

for any $X, Y \in \Gamma(T M)$.

Remark that the canonical distribution $D$ is $\varphi$-invariant since $D=\operatorname{Im} \varphi$. Also, the characteristic vector field $\xi$ is orthogonal to $D$ and therefore the tangent bundle splits orthogonally:

$$
T M=D \oplus\{\xi\}
$$

If the following condition is satisfied for an almost contact metric manifold $(M, \varphi, \xi, \eta, g)$, then it is called a Kenmotsu manifold

$$
\left(\nabla_{X} \varphi\right) Y=g(\varphi X, Y) \xi-\eta(Y) \varphi X
$$

where $\nabla$ is the Levi-Civita connection on $M$. For a Kenmotsu manifold, we also have

$$
\begin{aligned}
\nabla_{X} \xi & =X-\eta(X) \xi \\
R(X, Y) \xi & =\eta(X) Y-\eta(Y) X \\
R(X, \xi) Y & =g(X, Y) \xi-\eta(Y) X \\
S(X, \xi) & =-2 n \eta(X) \\
S(\xi, \xi) & =-2 n \\
Q \xi & =-2 n \xi
\end{aligned}
$$

where $S$ and $R$ are the Ricci tensor and Riemann curvature tensor of $M$, respectively and $Q$ is the Ricci operator defined by $S(X, Y)=g(Q X, Y)$.

Now, we recall some basic notions from [4], [18], [19], [23] as follows:

The projective curvature tensor $\mathcal{M}$, the extended projective curvature tensor $\mathcal{M}^{e}$ and the concircular curvature tensor $C$ of a $(2 n+1)$-dimensional manifold $(M, g)$ are defined by

$$
\begin{aligned}
M(X, Y) Z= & R(X, Y) Z-\frac{1}{4 n}\{S(Y, Z) X-S(X, Z) Y \\
& +g(Y, Z) Q X-g(X, Z) Q Y\} \quad(n \geq 1) \\
M^{e}(X, Y) Z= & M(X, Y) Z-\eta(X) M(\xi, Y) Z \\
& -\eta(Y) M(X, \xi) Z-\eta(Z) M(X, Y) \xi
\end{aligned}
$$

and

$$
C(X, Y) Z=R(X, Y) Z-\frac{r}{2 n(2 n+1)}\{g(Y, Z) X-g(X, Z) Y\}
$$


for any $X, Y, Z \in \Gamma(T M)$, where $r$ stands for the scalar curvature of $M$. From (2.11), (2.12), (2.13) and (2.15), we also have

$$
\begin{aligned}
M(X, Y) \xi= & \eta(X) Y-\eta(Y) X-\frac{1}{4 n}\{2 n \eta(X) Y-2 n \eta(Y) X \\
& +\eta(Y) Q X-\eta(X) Q Y\}, \\
R(Q X, Y) \xi= & -2 n \eta(X) Y-\eta(Y) Q X, \\
R(X, Q Y) \xi= & \eta(X) Q Y+2 n \eta(Y) X, \\
R(X, Y) Q \xi= & -2 n(\eta(X) Y-\eta(Y) X) .
\end{aligned}
$$

On the other hand, a Riemannian manifold $(M, g)$ is called $\eta$-Einstein if there exists two real constants $a$ and $b$ such that the Ricci tensor field $S$ of $M$ satisfies

$$
S=a g+b \eta \otimes \eta .
$$

Also, if the constant $b$ is equal to zero, then $M$ is called Einstein.

\section{Torse-forming Vector Field on Kenmotsu Manifold}

In this section, we deal with a Kenmotsu manifold $M$ endowed with a torseforming vector field $v$ and give some characterizations for such a vector field.

Now, we begin to this section with the following:

Proposition 3.1. Let $M$ be a Kenmotsu manifold endowed with a torse-forming vector field $v$. Then, the vector field $v$ is never on the distribution $D$ of $M$.

Proof. Let us assume that the vector field $v$ is on the distribution $D$. Then, using the fact that $g(v, \xi)=0$, we have

$$
g\left(\nabla_{X} v, \xi\right)+g\left(v, \nabla_{X} \xi\right)=0
$$

for any $X \in \Gamma(T M)$. Since the vector field $v$ is a torse-forming on $M$, from (1.2), (2.10) and (3.1), one has

$$
f \eta(X)+g(X, v)=0
$$

equivalently

$$
g(f \xi, X)=-g(X, v)
$$

Removing $X$ in the above equation gives

$$
v=-f \xi .
$$

This is a contradiction. Therefore, the vector field $v$ is never on distribution $D$. 
From (3.2), we can state the following corollary:

Corollary 3.1. Let $M$ be a Kenmotsu manifold endowed with a torse-forming vector field $v$. Then, $v$ is a pointwise collinear with the structure vector field $\xi$.

Theorem 3.1. Let $M$ be a Kenmotsu manifold endowed with a torse-forming vector field $v$ such that $v$ is a pointwise collinear with the structure vector field $\xi$. Then, we have the followings:

i) The vector field $v$ is a Killing on $M$.

ii) If $M$ admits a Ricci soliton with potential vector field $v$, then it is an expanding.

Proof. Let $v$ be a pointwise collinear with the structure vector field $\xi$. From (3.2), we write $v=-f \xi$. Then, we have

$$
\begin{aligned}
\nabla_{X} v & =\nabla_{X}(-f \xi) \\
& =-X(f) \xi-f \nabla_{X} \xi \\
& =-X(f) \xi-f(X-\eta(X) \xi)
\end{aligned}
$$

for any $X, Y \in \Gamma(T M)$. Since the vector field $v$ is a torse-forming on $M$, from equations (1.2), (3.2) and (3.3), one has

$$
-X(f) \xi-f(X-\eta(X) \xi)=f X-f \alpha(X) \xi .
$$

Also, taking the inner product of (3.4) with $\xi$ and using the equations (2.2), (2.7), we get

$$
X(f)=-f \eta(X)+f \alpha(X) .
$$

Again, taking the inner product of (3.4) with the arbitrary vector field $Y$ and using (2.2), (2.5) gives

$$
-X(f) \eta(Y)-f g(\varphi X, \varphi Y)=f g(X, Y)-f \alpha(X) \eta(Y) .
$$

By virtue of (2.5), (3.5) and (3.6), we find

$$
2 f g(\varphi X, \varphi Y)=0 \text {. }
$$

On the other hand, let $\left\{e_{1}, e_{2}, \ldots, e_{2 n}, e_{2 n+1}=\xi\right\}$ be an orthonormal basis of $T_{p} M, p \in M$. Putting $X=Y=e_{i}$ in (3.7) and summing over $i=1,2, \ldots, 2 n+1$, we obtain

$$
f=0 \text {. }
$$

From (3.2) and (3.8), we have $v=0$. As a result of this, the vector field $v$ is a Killing on $M$. Therefore, we write

$$
\left(£_{v} g\right)(X, Y)=0
$$


for any $X, Y \in \Gamma(T M)$.

Now, let us consider that $M$ admits a Ricci soliton with potential vector field $v$. Then, the equation (1.1) reduces to

$$
S(X, Y)=-\lambda g(X, Y)
$$

Putting $X=Y=\xi$ in (3.9) and using (2.13), we get $\lambda=2 n$. This shows that the Ricci soliton is expanding. Thus, the proof is completed.

Using the equation (3.8), we can give the following corollary:

Corollary 3.2. Let $M$ be a Kenmotsu manifold endowed with a torse-forming vector field $v$ such that $v$ is a pointwise collinear with the structure vector field $\xi$. Then, the vector field $v$ is never irrotational on $M$.

\section{Ricci Solitons and Some Curvature Conditions on Kenmotsu Manifold}

In this section, we give some important characterizations which classify a Kenmotsu manifold $M$ under some curvature conditions and study Ricci solitons on $M$.

The first result of this section is the following:

Theorem 4.1. Let $M$ be a Kenmotsu manifold admiting a Ricci soliton with the potential vector field $V$. If $V$ is orthogonal to $\xi$, then the Ricci soliton is expanding.

Proof. It follows immediately from the definition of Lie-derivative, we have

$$
\begin{aligned}
\left(£_{V} g\right)(\xi, \xi) & =g\left(\nabla_{\xi} V, \xi\right)+g\left(\nabla_{\xi} V, \xi\right) \\
& =2 g\left(\nabla_{\xi} V, \xi\right) .
\end{aligned}
$$

From the fact that $\nabla_{\xi} \xi=0$, it is easy to see that

$$
\nabla_{\xi}(g(V, \xi))=g\left(\nabla_{\xi} V, \xi\right)
$$

Since $M$ is a Ricci soliton, with the help of (1.1), (4.1) and (4.2), we get

$$
\begin{aligned}
S(\xi, \xi) & =-\frac{1}{2}\left(£_{V} g\right)(\xi, \xi)-\lambda g(\xi, \xi) \\
& =-g\left(\nabla_{\xi} V, \xi\right)-\lambda \\
& =-\nabla_{\xi}(g(V, \xi))-\lambda .
\end{aligned}
$$

Also, making use of (2.14) and (4.3), we find that

$$
\nabla_{\xi}(g(V, \xi))=2 n-\lambda .
$$


If the potential vector field $V$ is orthogonal to $\xi$, then the equation (4.4) becomes

$$
\lambda=2 n
$$

which shows that the Ricci soliton is expanding. Therefore, this completes the proof of the theorem.

The next example supports the Theorem 4.1 as follows:

Example 4.1. [12] We consider the three-dimensional Riemannian manifold $M=\{(x, y, z) \in$ $\left.\mathbb{R}^{3},(x, y, z) \neq(0,0,0)\right\}$ and the linearly independent vector fields

$$
e_{1}=z \frac{\partial}{\partial x}, \quad e_{2}=z \frac{\partial}{\partial y}, \quad e_{3}=-z \frac{\partial}{\partial z},
$$

where $(x, y, z)$ are the Cartesian coordinates in $\mathbb{R}^{3}$. Let $g$ be the Riemannian metric defined by

$$
\begin{aligned}
& g\left(e_{i}, e_{i}\right)=1 \\
& g\left(e_{i}, e_{j}\right)=0 \text { for } \quad i \neq j .
\end{aligned}
$$

and is given by

$$
g=\frac{1}{z^{2}}\{d x \otimes d x+d y \otimes d y+d z \otimes d z\} .
$$

Also, let $\eta, \varphi$ be the $1-$ form and the $(1,1)$-tensor field, respectively defined by

$$
\eta(Z)=g\left(Z, e_{3}\right), \quad \varphi\left(e_{1}\right)=-e_{2}, \quad \varphi\left(e_{2}\right)=e_{1}, \quad \varphi\left(e_{3}\right)=0
$$

for any $Z \in \Gamma(T M)$. Hence, $(M, \varphi, \xi, \eta, g)$ becomes an almost contact metric manifold with the characteristic vector field $e_{3}=\xi$.

By direct calculations, we have

$$
\left[e_{1}, e_{2}\right]=0, \quad\left[e_{1}, e_{3}\right]=e_{1} \text { and }\left[e_{2}, e_{3}\right]=e_{2} .
$$

On the other hand, using Koszul's formula for the Riemannian metric $g$, we get:

$$
\nabla_{e_{1}} e_{3}=e_{1}, \quad \nabla_{e_{2}} e_{3}=e_{2}, \quad \nabla_{e_{3}} e_{3}=0
$$

and others

$$
\nabla_{e_{1}} e_{2}=\nabla_{e_{2}} e_{1}=\nabla_{e_{3}} e_{1}=\nabla_{e_{3}} e_{2}=0, \quad \nabla_{e_{1}} e_{1}=\nabla_{e_{2}} e_{2}=-e_{3} .
$$

Therefore, the manifold $M$ is a 3 -dimensional Kenmotsu manifold. Using the equations (4.5) and (4.6), we find

$$
\begin{array}{lll}
R\left(e_{1}, e_{2}\right) e_{3}=0, & R\left(e_{1}, e_{3}\right) e_{2}=0, & R\left(e_{2}, e_{3}\right) e_{1}=0, \\
R\left(e_{1}, e_{2}\right) e_{2}=-e_{1}, & R\left(e_{1}, e_{2}\right) e_{1}=e_{2}, & R\left(e_{1}, e_{3}\right) e_{3}=-e_{1}, \\
R\left(e_{1}, e_{3}\right) e_{1}=e_{3}, & R\left(e_{2}, e_{3}\right) e_{3}=-e_{2}, & R\left(e_{3}, e_{2}\right) e_{2}=-e_{3},
\end{array}
$$

which yields

(4.7) $S\left(e_{1}, e_{1}\right)=-2, \quad S\left(e_{2}, e_{2}\right)=-2, \quad S\left(e_{3}, e_{3}\right)=-2 \quad$ and $\quad S\left(e_{i}, e_{j}\right)=0$

for all $i, j=1,2,3(i \neq j)$. In this case, the manifold $M$ is a Ricci soliton with potential vector field $e_{1}$ or $e_{2}$ which satisifes the equation (1.1) for $\lambda=2$. 
Theorem 4.2. Let $M$ be a Kenmotsu manifold such that the condition $Q . \mathcal{M}=0$ is satisfied. Then, $M$ is an Einstein manifold.

Proof. Suppose that $M$ satisfies the condition $(Q \cdot \mathcal{M})(X, Y) Z=0$, namely,

$$
Q(\mathcal{M}(X, Y) Z)-\mathcal{M}(Q X, Y) Z-\mathcal{M}(X, Q Y) Z-\mathcal{M}(X, Y) Q Z=0
$$

for any $X, Y, Z \in \Gamma(T M)$, where $Q$ stands for the Ricci operator defined by $S(X, Y)=$ $g(Q X, Y)$. Putting $Z=\xi$ in (4.8) gives

(4.9) $Q(\mathcal{M}(X, Y) \xi)-\mathcal{M}(Q X, Y) \xi-\mathcal{M}(X, Q Y) \xi-\mathcal{M}(X, Y) Q \xi=0$.

For the first and second term of (4.9), if we use (2.13), (2.15), (2.19) and (2.20), we get

$$
\begin{aligned}
Q(M(X, Y) \xi)= & \eta(X) Q Y-\eta(Y) Q X-\frac{1}{4 n}\{2 n \eta(X) Q Y-2 n \eta(Y) Q X \\
& \left.+\eta(Y) Q^{2} X-\eta(X) Q^{2} Y\right\} \\
\mathcal{M}(Q X, Y) \xi= & -2 n \eta(X) Y-\eta(Y) Q X-\frac{1}{4 n}\left\{-4 n^{2} \eta(X) Y-2 n \eta(Y) Q X\right. \\
& \left.+2 n \eta(X) Q Y+\eta(Y) Q^{2} X\right\} .
\end{aligned}
$$

For the third and fourth term of (4.9), making use of (2.13), (2.15), (2.21) and (2.22), we derive

$$
\begin{aligned}
M(X, Q Y) \xi= & \eta(X) Q Y+2 n \eta(Y) X-\frac{1}{4 n}\left\{4 n^{2} \eta(Y) X+2 n \eta(X) Q Y\right. \\
& \left.-2 n \eta(Y) Q X-\eta(X) Q^{2} Y\right\} \\
\mathcal{M}(X, Y) Q \xi= & -2 n(\eta(X) Y-\eta(Y) X)-\frac{1}{4 n}\left\{4 n^{2} \eta(Y) X-4 n^{2} \eta(X) Y\right. \\
& -2 n \eta(Y) Q X+2 n \eta(X) Q Y\} .
\end{aligned}
$$

If we substitute (4.10)-(4.13) in (4.9), after some calculations we obtain

$$
2 n \eta(X) Y-2 n \eta(Y) X+\eta(X) Q Y-\eta(Y) Q X=0 .
$$

Putting $Y=\xi$ in (4.14) and using the equalities (2.2), (2.15) yields

$$
Q X=-2 n X
$$

Taking the inner product of (4.15) with $W$, we have

$$
S(X, W)=-2 n g(X, W)
$$

for any $W \in \Gamma(T M)$. This completes the proof of the theorem.

Using the equality (4.16), we can give the following corollary. 
Corollary 4.1. Let $M$ be a Kenmotsu manifold such that the condition $Q . \mathcal{M}=0$ is satisfied. If $M$ admits a Ricci soliton with the potential vector field $\xi$, then the Ricci soliton is expanding.

Proof. It follows from the definition of the Lie-derivative and from (2.10), we have

$$
\begin{aligned}
\left(£_{\xi} g\right)(X, Y) & =g\left(\nabla_{X} \xi, Y\right)+g\left(\nabla_{Y} \xi, X\right) \\
& =g(X-\eta(X) \xi, Y)+g(Y-\eta(Y) \xi, X) \\
& =2 g(X, Y)-2 \eta(X) \eta(Y)
\end{aligned}
$$

for any $X, Y \in \Gamma(T M)$. Since $M$ is a Ricci soliton, from (1.1) we write

$$
\left(£_{\xi} g\right)(X, Y)+2 S(X, Y)+2 \lambda g(X, Y)=0 .
$$

If we use the equalities (4.16) and (4.17) in (4.18), we get

$$
(2-4 n+2 \lambda) g(X, Y)-2 \eta(X) \eta(Y)=0 .
$$

Putting $X=Y=\xi$ in (4.19) and using (2.2) gives $\lambda=2 n$ which means that the Ricci soliton is expanding. This result ends the proof of the corollary.

Theorem 4.3. Let $M$ be a Kenmotsu manifold such that the condition $C . Q=0$ is satisfied. Then, $M$ is either of constant scalar curvature or $M$ is an Einstein manifold.

Proof. Let us suppose that the manifold satisfies the condition $(C(X, Y) \cdot Q) Z=0$, that is,

$$
C(X, Y) Q Z-Q(C(X, Y) Z)=0
$$

for any $X, Y, Z \in \Gamma(T M)$. Substituting $Y=\xi$ in (4.20), one has

$$
C(X, \xi) Q Z-Q(C(X, \xi) Z)=0 .
$$

Futhermore, with the help of (2.7) and (2.12), we get

$$
C(X, \xi) Z=\left(1+\frac{r}{2 n(2 n+1)}\right)(g(X, Z) \xi-\eta(Z) X) .
$$

Replacing $Z$ by $Q Z$ in (4.22) and using (2.7), (2.13) we have

$$
C(X, \xi) Q Z=\left(1+\frac{r}{2 n(2 n+1)}\right)(S(X, Z) \xi+2 n \eta(Z) X) .
$$

Applying $Q$ to the both sides of (4.22) and from (2.15), we infer

$$
Q(C(X, \xi) Z)=\left(1+\frac{r}{2 n(2 n+1)}\right)(-2 n g(X, Z) \xi-\eta(Z) Q X) .
$$


From (4.21), (4.23) and (4.24), we write

$$
\left(1+\frac{r}{2 n(2 n+1)}\right)(S(X, Z) \xi+2 n \eta(Z) X+2 n g(X, Z) \xi+\eta(Z) Q X)=0 .
$$

Taking the inner product of the above equation with $\xi$ and making use of (2.7), (2.13), we have

$$
\left(1+\frac{r}{2 n(2 n+1)}\right)(S(X, Z)+2 n g(X, Z))=0
$$

which implies that

$$
r=-2 n(2 n+1)
$$

or

$$
S(X, Z)=-2 n g(X, Z)
$$

This is the desired result. Thus, the proof is completed.

Theorem 4.4. Let $M$ be a Kenmotsu manifold with vanishing extended $\mathcal{M}^{e}$-projective curvature tensor. Then, the followings are satisfied:

i) $M$ is an Einstein manifold.

ii) $M$ is locally isometric to the hyperbolic space $H^{(2 n+1)}(-1)$ if and only if $\mathcal{M}$-projective curvature tensor vanishes.

iii) If $M$ admits a Ricci soliton with the potential vector field $V$, then $V$ is a conformal Killing on $M$.

Proof. Let $M$ be a Kenmotsu manifold with vanishing extended $\mathcal{M}^{e}$-projective curvature tensor. Then, the equation (2.17) becomes

(4.25) $M(X, Y) Z=\eta(X) M(\xi, Y) Z+\eta(Y) M(X, \xi) Z+\eta(Z) M(X, Y) \xi$

for any $X, Y, Z \in \Gamma(T M)$. If we take $X=\xi$ in (4.25), we have

$$
\eta(Y) M(\xi, \xi) Z+\eta(Z) M(\xi, Y) \xi=0
$$

From the equalities (2.11)-(2.16), we get

$$
M(\xi, \xi) Z=0
$$

and

$$
M(\xi, Y) \xi=Y-\eta(Y) \xi-\frac{1}{4 n}(-4 n \eta(Y) \xi+2 n Y-Q Y)
$$


Using (4.27) and (4.28) in (4.26) and after simple calculations, one has

$$
\left.\eta(Z) Y-\frac{1}{2} \eta(Z) Y+-\frac{1}{4 n} \eta(Z) Q Y\right)=0 .
$$

Substituting $Z=\xi$ in (4.29), then the equation (4.29) is reduced to

$$
Q Y=-2 n Y .
$$

Also, taking the inner product of (4.30) with $W$, we have

$$
S(Y, W)=-2 n g(Y, W) .
$$

for any $W \in \Gamma(T M)$. Therefore, $M$ is an Einstein manifold. Making use of (4.30) and (4.31) in (2.16) gives

$$
M(X, Y) Z=R(X, Y) Z-\frac{1}{4 n}\{-4 n g(Y, Z) X+4 n g(X, Z) Y\},
$$

that is,

$$
M(X, Y) Z=R(X, Y) Z+\{g(Y, Z) X-g(X, Z) Y\} .
$$

This proves $i i)$.

On the other hand, let us consider that $M$ is a Ricci soliton with the potential vector field $V$. Then, from (1.1) and (4.31) we conclude that

$$
\begin{aligned}
\left(£_{V} g\right)(X, Y) & =-2 S(X, Y)-2 \lambda g(X, Y) \\
& =(4 n-2 \lambda) g(X, Y)
\end{aligned}
$$

which implies that the potential vector field $V$ is a conformal Killing on $M$. Consequently, we get the requested results.

\section{REF EREN C E S}

1. G. Ayar, M. Yildirim: Ricci Solitons and Gradient Ricci Solitons on Nearly Kenmotsu Manifolds. Facta Univ. Ser. Math. Inform. 34 (3) (2019), 503-510.

2. A. M. Blaga, M. Crasmareanu: Torse-forming $\eta$-Ricci Solitons in Almost Paracontact $\eta$ - Einstein Geometry. Filomat. 31 (2) (2017), 499-504.

3. D. E. Blair: Contact Manifolds in Riemannian Geometry, Lecture Notes in Mathematics, 509, Springer-Verlag, Berlin, 1976.

4. D. E. Blair, J. S. Kim, M. M. Tripathi : On the Concircular Curvature Tensor of a Contact Metric Manifold. J. Korean Math. Soc. 42 (5) (2005), 883-992.

5. B.-Y. Chen: Classification of Torqued Vector Fields and Its Applications to Ricci Solitons. Kragujevac J. Math. 41 (2) (2017), 239-250. 
6. B.-Y. Chen : Some Results on Concircular Vector Fields and Their Applications to Ricci Solitons. Bull. Korean Math. Soc. 52 (5) (2015), 1535-1547.

7. J. T. Cho, R. Sharma: Contact Geometry and Ricci Solitons. Int. J. Geom. Methods Mod. Phys. 7 (6) (2010), 951-960.

8. M. Crasmareanu: Scalar Curvature for Middle Planes in Odd-Dimensional Torse-forming Amost Ricci Solitons. Kragujevac J. Math. 43 (2) (2019), 275279.

9. A. Ghosh: Kenmotsu 3-Metric as a Ricci Soliton. Chaos, Solitons \& Fractals 44 (8) (2011), 647-650.

10. A. Ghosh: Ricci Soliton and Ricci Almost Soliton within the Framework of Kenmotsu Manifold. Carpathian Math. Publ. 11 (1) (2019), 56-69.

11. R. S. Hamilton: The Ricci Flow on Surfaces, Mathematics and General Relativity (Santa Cruz, CA, 1986). Contemp. Math. A.M.S. 71 (1988), 237-262.

12. S. K. Hui, S. K. Yadav, A. Patra: Almost Conformal Ricci Solitons on f-Kenmotsu Manifolds. Khayyam J. Math. 5 (1) (2019) 89-104.

13. K. Kenmotsu: A Class of Almost Contact Riemannian Manifolds. Tohoku Math. J. 24 (1972), 93-103.

14. Y. C. Mandal, S. K. HuI: Yamabe Solitons with Potential Vector Field as Torse-forming. CUBO 20 (3) (2018), 37-47.

15. Ş. E. Meriç, E. KILIÇ: Riemannian Submersions Whose Total Manifolds Admit a Ricci Soliton. Int. J. Geom. Methods Mod. Phys. 16 (12) (2019), 1950196.

16. A. Minai, I. MinAi: Torse forming Vector Fields and Exterior Concurrent Vector Fields on Riemannian Manifolds and Applications. J. Geom. Phys. 73 (2013), 200-208.

17. D. S. PAtra: Ricci Solitons and Ricci Almost Solitons on Para-Kenmotsu Manifold. Bull. Korean Math. Soc. 56 (5) (2019), 1315-1325.

18. G. P. Pokhariyal, R. S. Mishra: Curvature Tensor and Their Relavistic Significance II. Yokohama Math. J. 19 (1971) 97-103.

19. D. G. Prakasha, K. Mirji: On the $\mathcal{M}$-Projective Curvature Tensor of a $(k, \mu)$-Contact Metric Manifold. Facta Univ. Ser. Math. Inform. 32 (1) (2017), $117-128$

20. R. Sharma: Certain Results on K-Contact and $(k, \mu)$-Contact Manifolds. J. Geom. 89 (2008), 138-147.

21. Y. Wang, X. Liu: Ricci Solitons on Three-Dimensional $\eta$-Einstein Almost Kenmotsu Manifolds. Taiwanese J. Math. 19 (1) (2015), 91-100.

22. K. Yano: On Torse-forming Direction in a Riemannian Space. Proc. Imp. Acad. Tokyo, 20 (1944), 340-345.

23. K. Yano, M. Kon: Structures on Manifolds. Series in Mathematics, World Scientific Publishing, Springer, 1984.

24. H. İ. Yoldaş, Ş. E. Meriç, E. YAŞAR: On Generic Submanifold of Sasakian Manifold with Concurrent Vector Field. Commun. Fac. Sci. Univ. Ank. Ser. A1 Math. Stat. 68 (2) (2019), 1983-1994.

25. H. İ. YoldAŞ, Ş. E. MeRIÇ, E. YAŞAR: On submanifolds of Kenmotsu manifold with Torqued vector field. Hacettepe Journal of Mathematics and Statistics, 49 (2) (2020), 843-853. 
Halil İbrahim Yoldaş

Faculty of Science and Arts

Department of Mathematics

Mersin University

33343 Mersin, Turkey

hibrahimyoldas@gmail.com

Erol Yaşar

Faculty of Science and Arts

Department of Mathematics

Mersin University

33343 Mersin, Turkey

eroly69@gmail.com 